\title{
Gastric Intraepithelial Neoplasia
}

National Cancer Institute

\section{Source}

National Cancer Institute. Gastric Intraepithelial Neoplasia. NCI Thesaurus. Code C3955.

A intraepithelial neoplasia (dysplasia) that arises in either the native gastric mucosa or in metaplastic gastric epithelium. It has a flat, polypoid or slightly depressed growth pattern.

It is graded as low grade (mild dysplasia) or high grade (moderate dysplasia and carcinoma in situ). (WHO, 2000) 